\title{
Source and Drain Junction Engineering for Enhanced Non-Volatile Memory Performance
}

\author{
Sung-Jin Choi and Yang-Kyu Choi \\ Department of Electrical Engineering, KAIST \\ Republic of Korea
}

\section{Introduction}

There is strong demand to maintain the trend of increasing bit density and reducing bit cost in Flash memory technology. To this end, aggressive scaling of the device dimension and multi-level cell (MLC) or multi-bit cell (MBC) have been proposed in NAND and NOR Flash memory architectures. However, especially in NAND Flash memory, bit cost is expected to rise in the near future, because the process cost will increase more rapidly than the shrink rate. One solution to avoid such challenges is the use of three dimensionally stacked array structures, based on polycrystalline silicon (poly-Si). The utilization of poly-Si in the channel not only increases pass disturbs but also reduces the worst case string current. Indeed, for every doubling in density, the worst case string current halves. Since the channel of these devices is poly-Si and source/drain (S/D) regions are not formed (i.e., a junction-free structure), the worst case string current (all cells in a string with high threshold voltage $\left(\mathrm{V}_{\mathrm{T}}\right)$ ) will quickly tend toward unreadably low values as density increases (Walker, 2009). Therefore, it is worthwhile to note that the impact on the S/D structures becomes important. Moreover, Fowler-Nordheim (FN) tunneling for programming is still very slow for certain applications that require high-speed operation.

In NOR Flash memory, channel length scaling has threatened continued scaling and approaching its end point. For uniform channel hot electron injection (CHEI) programming, a robust margin for punch-through is a pre-requisite for cell transistors. However, CHEI programming aggravates immunity against punch-through by increasing the drain voltage to a level that will trigger CHEI. It is clear that the drain voltage window to guarantee both programming speed and margin from drain disturbance is narrowed as the channel length scales down. Moreover, the low injection efficiency compromising from vertical and lateral fields and the high parasitic resistance at the S/D junctions also impose a constraint on scaling the cell size reduction. Consequently, the lower effective program voltage due to the high parasitic S/D resistance in an extremely scaled cell results in a small $\mathrm{V}_{\mathrm{T}}$ window and thereafter retards the program speed.

Herein S/D engineering for enhanced performance of Flash memory for two novel structures is demonstrated: (i) a dopant-segregated Schottky-barrier device (DSSB), and (ii) a junctionless MOSFET. First, we utilized dopant-segregated metallic silicide S/D junctions on charge trapping memory cells. They boosted the program speed even at a low program bias with the aid of abrupt band bending at the edge of metal silicided junctions. Second, the 
structure of the junctionless transistor was examined from S/D junction engineering and cell size scaling points of view.

\section{Schottky-Barrier (SB) MOSFET}

SB-MOSFETs were initially proposed by Lepselter and Sze four decades ago (1968 - Bell Labs.), shortly after the invention of the current type of MOSFET by Kahng and Attala (1960 - Bell Labs.). Being different from the conventional MOSFET with doped/diffused S/D junctions, the SB-MOSFET has metallic silicided S/D junctions, realizing by employing a self-aligned silicide process, as shown in Fig. 2-1. The operating principle is based on gate induced electronic band bending to modulate the S/D thermionic and tunneling barrier (Larson et al., 2006). One remarkable advantage of the SB-MOSFETs is their low interface contact resistivity: $\rho_{\mathrm{c}} \sim 10^{-9} \Omega \cdot \mathrm{cm}^{2}$ for metallic S/D compared with $\rho_{\mathrm{c}} \sim 10^{-7} \Omega \cdot \mathrm{cm}^{2}$ in standard doped S/D junctions. Moreover, it is easier to control the abruptness/shallowness of the S/D junctions in metallic S/D junctions than in standard doped S/D junctions, and the solid solubility limitation associated with doping can also be resolved. From a fabrication viewpoint, the silicidation process is fully compatible with the standard CMOS technologies and does not require a high temperature annealing process; this prevents thermal degradation (in particular, for high-k gate dielectric layers and metal-gates) and reduces fabrication costs. However, for typical SB-MOSFETs, the on-state current is significantly limited by the existence of a SB height (SBH) at the S/D junctions; thus, the performance of SB-MOSFETs is still not comparable with that of conventional MOSFETs with highly doped S/D junctions. Therefore, it is necessary to find an appropriate material with a low SBH and develop a method to reduce the effective SBH, such as a dopantsegregation technique (Kinoshita et al., 2004), in order to enhance the performance of SBMOSFETs.

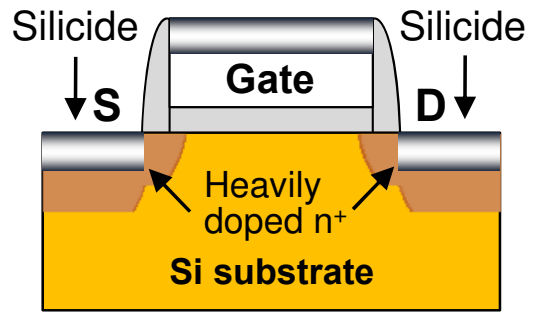

(a)

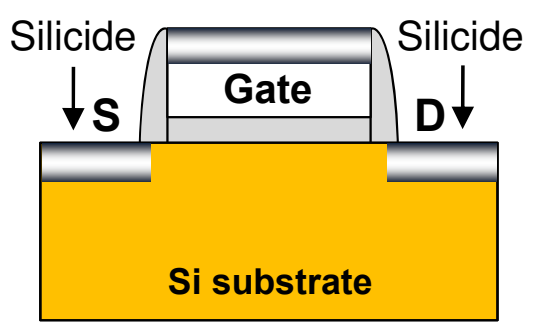

(b)

Fig. 2-1. Simplified schematic of (a) the conventional and (b) the SB devices

SB-MOSFETs are also interesting devices from a physics perspective. They can be used for high speed devices in highly scaled regimes because they have an abrupt energy band bending, which results from a large voltage drop at the source to the inversion channel. Importantly, a high lateral electric field exists around not the drain but the source edge. The carriers, e.g., electrons for an n-channel SB-MOSFET, injected from the source thermally or via tunneling are accelerated by this electric field and become hot around the source edge. These properties are very useful and interesting for both logic and memory devices. 


\subsection{Operating principle of SB-MOSFETs}

A band diagram schematically depicting the different operations of SB-MOSFETs is shown in Fig. 2-2. A small off-state current is possible at a gate voltage of $0 \mathrm{~V}$ as a result of the high effective barrier height for both electrons and holes. The effective barrier height for holes at a gate voltage of $0 \mathrm{~V}$ consists of two components: the intrinsic barrier for holes $\left(\Phi_{\mathrm{bp}}\right)$ and the contact barrier $\left(\Phi_{\text {contact }}\right)$. The contact potential results from the built-in potential energy $\left(\psi_{\mathrm{b}}\right)$ arising from the metal-semiconductor interface and the surface potential energy $\left(\psi_{\mathrm{s}}\right)$ resulting from the gate $\left(\Phi_{\text {contact }}=\psi_{\mathrm{b}}+\psi_{\mathrm{s}}\right)$ (Fig. 2-2(a)). As a negative gate bias is applied, the effective barrier for holes is reduced to the intrinsic barrier height $\left(\Phi_{\mathrm{bp}}\right)$ and the current increases as holes are ejected over the barrier primarily via thermionic emission (Fig. 2-2(b)). Note that when the effective barrier height $\left(\Phi_{\text {effective }}=\Phi_{\mathrm{bp}}+\Phi_{\text {contact }}\right)$ is the same as the intrinsic barrier height, the flat band source-to-body condition is formed (i.e., $\Phi_{\text {contact }} \sim 0 \mathrm{eV}$ ). For small gate voltage values, the drain voltage will mostly drop at the source, i.e., the reverse bias contact, and current transport can be performed by the thermionic emission. This also holds in the subthreshold regime (small gate voltage). Further increases of the gate bias cause the bands to bend upwards and holes to tunnel through the barrier either directly or with thermal assistance (Fig. 2-2(c)). As noted above, the SBH limits the current flow in the subthreshold regime and becomes conductive in the on-state, where the channel resistance limits the current flow in an ideal case.

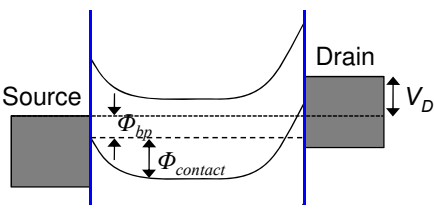

(a)

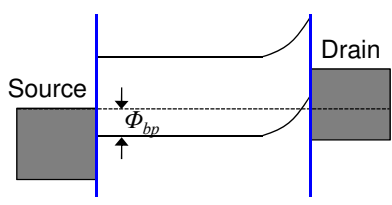

(b)

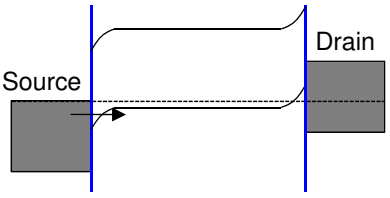

(c)

Fig. 2-2. Band diagrams of the different operating regimes of an SB-MOSFET: (a) off-state, (b) subthreshold regime, and (c) on-state.

\subsection{Dopant-segregation technique}

As mentioned earlier, the SB can limit the current drivability if an appropriate low SB material is not used. The dopant-segregation technique, an attractive technique to enhance the current density, has been introduced to SB-MOSFETs. If silicidation is performed on the doped silicon regions, the dopants can be redistributed at the interface between the silicide and silicon, which significantly affects the electrostatic properties of the SB junctions (Muraka, S. P. et al., 1087). The redistribution of dopants is determined by the diffusivity and solid solubility of dopants in the silicide and the presence of point defects at the interface between the silicide and silicon. Thermal annealing of silicide materials on ion implanted or doped (i.e., activated or non-activated) silicon can induce redistribution of dopants during the silicidation process. In particular, dopants are segregated at the interface between the silicide and silicon as a result of the different solid solubility of these materials. Atoms of nickel (Ni), a candidate material in SB-MOSFETs, are the moving species, supplied by diffusion through the growing silicide layer to the silicide/Si interface. Subsequently, the covalent bonding of $\mathrm{Si}$ atoms is softened by the diffusion of $\mathrm{Ni}$ atoms. A significant change of volume occurs when the silicide is formed, which leads to high strain at the interface. As 
a result, point defects (self-interstitial or vacancies) can be generated to partially relieve the stress. Due to the formation of vacancies, the diffusivity of the arsenic in the silicon is enhanced and it is forced out of the silicon after the silicide is formed. The arsenic dopants move towards the interface where they accumulates at the moving interface between the silicide and silicon. Although the dopant concentrations are generally below the solid solubility limit in the silicides and silicon, the point defects induced by the high strain interface can lead to the increment of local dopant concentrations that are higher than the solid solubility. This segregation of dopants is possible with boron, antimony, sulfur, chlorine, etc. as well as arsenic. The segregated dopants form a thin layer with a high concentration, which causes strong upward or downward band bending depending on the type of dopants, as shown in Fig. 2-3. As a consequence, the tunneling probability of the carriers through the effectively lowered Schottky barrier increases significantly. Although dopant-segregation is performed at relatively low temperatures, a fraction of the dopants is located at substitution sites in the silicon lattice and is therefore activated. Therefore, owing to enhanced injection efficiency, dopant-segregated SB (DSSB) MOSFETs have attracted considerable attention as a candidate for a high performance devices in future ULSIs.

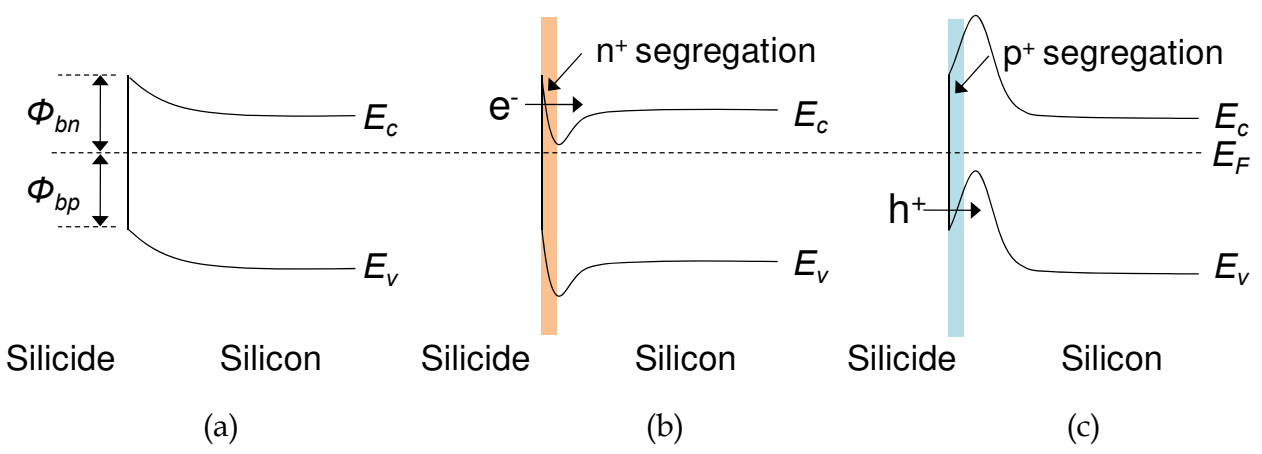

Fig. 2-3. Schematic band diagrams of (a) a mid-gap silicide with equal SB-heights for electrons and holes, (b) band bending induced by segregated n-type dopants, and (c) band bending induced by segregated p-type dopants.

\subsection{Application to non-volatile memory devices}

The metal-semiconductor SB diode is also known as a 'hot carrier diode'. The injected carriers from the semiconductor to the metal electrode, regardless of whether the injection mechanism is thermionic emission or tunneling, are forward biased Schottky barrier diodes, and they can obtain higher energies than the Fermi energies at the metal side. Moreover, the carriers injected from the metal to the semiconductor in the reverse biased junction can obtain higher energies than the Fermi energies in the semiconductor side, as shown in Fig. 2-4.

Both SB and DSSB MOSFETs at the on-state have an abrupt lateral voltage drop at the source end of the device due to the reverse biased source Schottky diode (Uchida et al., 2000, Kinoshita et al., 2006); therefore, a natural high electric field exists around the source edge. The carriers injected from the source electrode thermally or by tunneling will be accelerated by this electric field and will become hot around the source edge. 


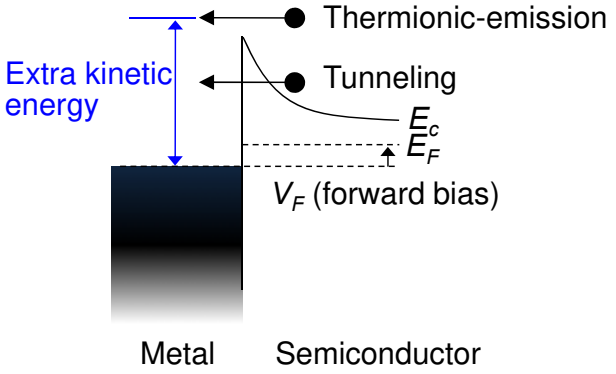

(a)

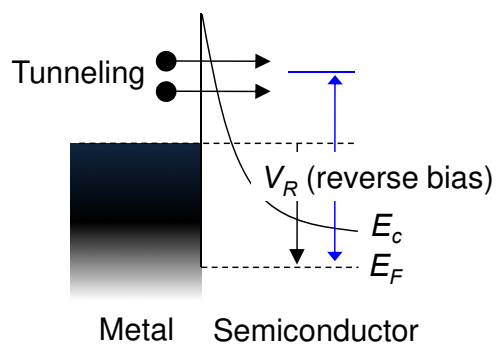

(b)

Fig. 2-4. (a) Conceptual explanation of hot carriers in (a) forward biased and (b) reverse biased in Schottky barrier diodes.

\section{SB Flash memory}

\subsection{Hot-carrier program in double-gate DSSB FinFETs}

Gate length scaling is the most critical limit in a NOR Flash memory cell, which uses a program method known as CHEI. This method aggravates immunity against punchthrough by increasing the drain voltage to a level that can trigger CHEI, as shown in Fig. 3-1(a). In addition, the low injection efficiency of the hot electrons generated at the drain side and the high parasitic resistance at the S/D also impose a constraint on scaling the cell size down. Consequently, the lower effective program voltage due to the high parasitic S/D resistance in an extremely scaled cell results in a small $\mathrm{V}_{\mathrm{T}}$ window and thereupon retards the program speed. CHEI programming in conventional NOR-type Flash memories also poses a constraint on the choice of the proper gate voltage $\left(\mathrm{V}_{\mathrm{G}}\right)$ and drain voltage $\left(\mathrm{V}_{\mathrm{D}}\right)$, as shown in Fig. 3-1(b). A high $V_{D}$ is necessary to induce a high lateral electric field for the generation of hot electrons. Furthermore, a high $\mathrm{V}_{\mathrm{G}}$ is indispensable for attaining a sufficient vertical electric field for the injection of hot electrons into a charge storage node. Simultaneous optimization of the lateral and vertical electric fields is very difficult. Moreover, the high voltage needed to generate an adequate amount of hot electrons for programming consumes a large amount of power.

The source-side injection of hot electrons for programming at low voltage is therefore attractive because of its high injection efficiency and the absence of constraints on the cooptimization of $V_{G}$ and $V_{D}$. Previous reports on source-side injection by the decoupling of hot electrons from the drain field demonstrated a fast low-voltage programming operation (Wu et al., 1986); however, it is difficult to adapt this approach to NOR-type Flash memory as it requires extra processes and different circuitry. In this section, an intensive analysis of NOR Flash memory, where double-gate (DG) DSSB FinFET silicon-oxide-nitride-oxidesilicon (SONOS) devices are employed, is carried out. The program speed is boosted even at a low program bias owing to the improved CHEI, which is enabled by the inherent sharp band bending of the DSSB at the source side. The DSSB structure provides several benefits, including increased lateral and vertical fields, excellent injection efficiency into the charge storage node, and a drain disturbance-free feature against a conventional device composed of diffused p-n junctions. 


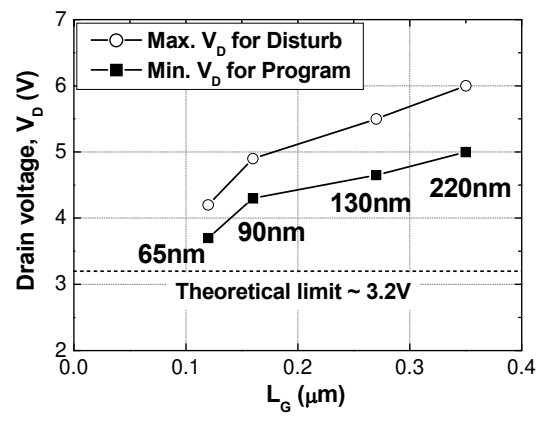

(a)

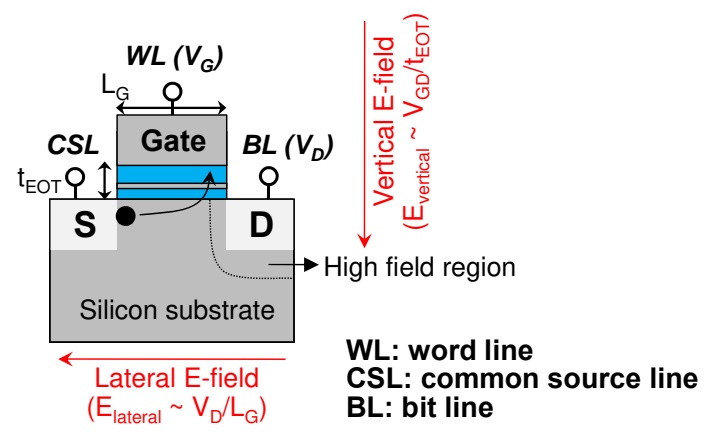

(b)

Fig. 3-1. (a) Scaling trend of drain biases. Minimum bias for programming speed and maximum bias for allowable drain disturbance are drawn for NOR flash generations. (b) Trade-off relations between vertical field and lateral field in the conventional CHEI programming method.

\subsubsection{Device fabrication}

The process schematics and sequences are summarized in Fig. 3-2. The process flow of the DSSB FinFET SONOS device is the same as that of the conventional FinFET except for the formation of gate spacers and the silicided S/D junctions. Using a shallow implantation of arsenic (As) after the formation of gate spacers, the SB height is effectively modulated by using segregated dopants. During the formation of the gate spacers, the S/D regions are recessed so that they subsequently provide a uniform S/D along the fin depth (vertical direction). This task is challenging with only S/D implantation and activation. Finally, the DSSB S/D was formed by means of nickel silicidation (NiSi) in a two-step rapid thermal processing (RTP), which can minimize the lateral diffusion of NiSi.

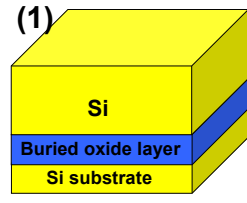

(3)

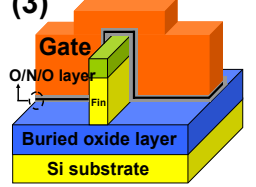

(a)
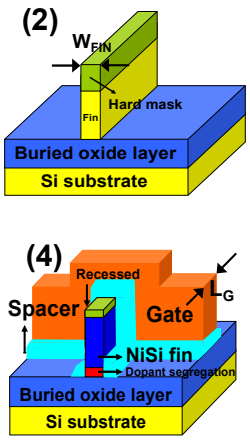

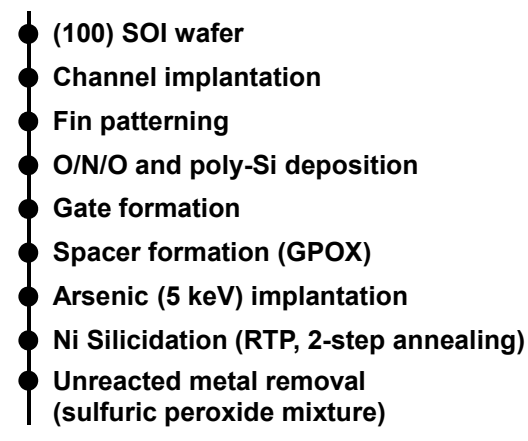

(b)

Fig. 3-2. Flow chart of the DSSB FinFET SONOS device. In the silicidation process, a twostep RTP is used to reduce any overgrowth of NiSi and mitigate lateral diffusion. Since the SB height is effectively modulated by the dopant concentration, a shallow implantation (5 $\mathrm{keV}$ ) of As was applied. 


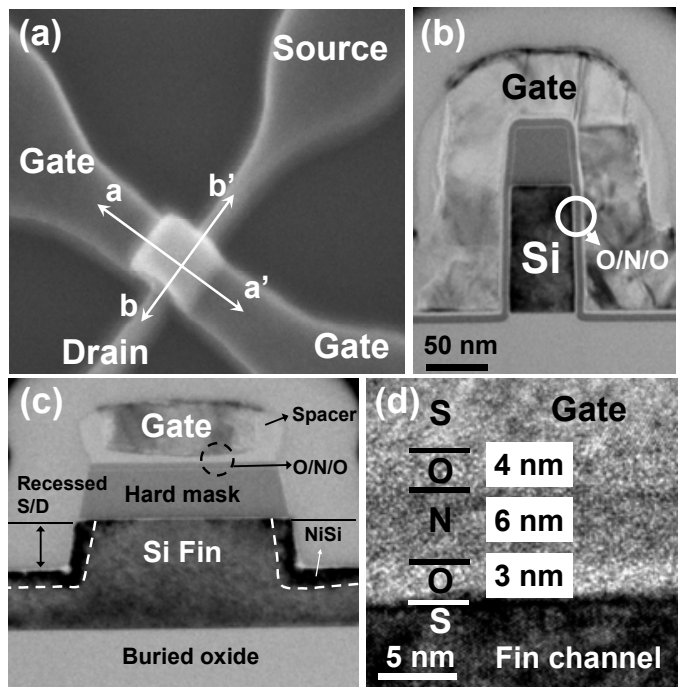

Fig. 3-3. SEM and TEM images of the fabricated devices (Choi et al., 2008).

The SEM photograph in Fig. 3-3(a) shows a bird's-eye view of the fabricated DSSB FinFET SONOS device. Fig. 3-3(b), 3-3(c), and 3-3(d) are cross-sectional TEM images from various points of view of the DSSB FinFET SONOS device. The device has a gate length of $220 \mathrm{~nm}$ and a fin that ranges in width from $30 \mathrm{~nm}$ to $100 \mathrm{~nm}$. For the control group, a conventional FinFET SONOS device with a diffused p-n junction was also fabricated. In Fig. 3-4, the results obtained from a scanning TEM image for verification of dopant segregation are shown. Dopant segregation at the interface between the silicon and the silicide is clearly observed.
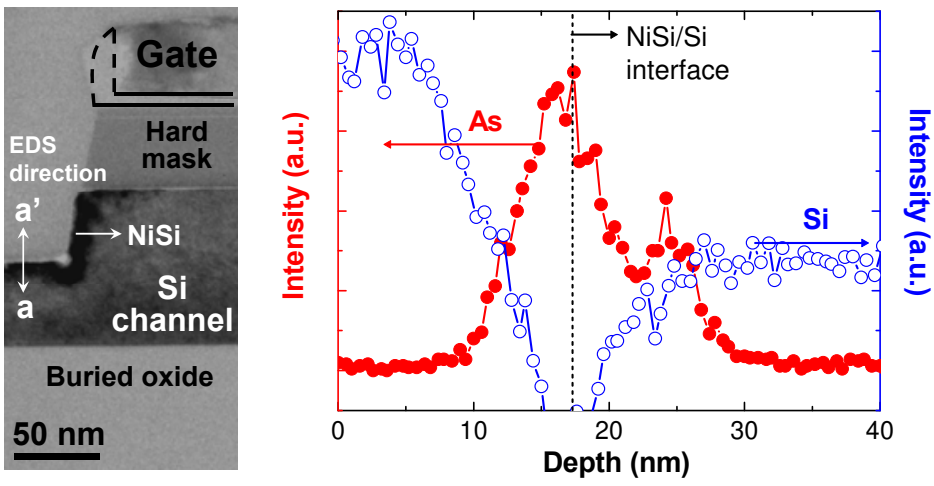

Fig. 3-4. TEM image of DG DSSB SONOS and STEM energy dispersive spectromotry (EDS) analysis. (Choi et al., 2009a)

\subsubsection{Memory characteristics}

Fig. 3-5(a) schematically illustrates the different injection mechanism of hot electrons for the DSSB Flash memory device and the conventional Flash memory device under the 
programming bias condition of $\mathrm{CHEI}\left(\mathrm{V}_{\mathrm{G}}>0\right.$ and $\left.\mathrm{V}_{\mathrm{D}}>0\right)$. In the case of the conventional Flash memory device, hot electrons are generated near the drain-side where the device is under a high lateral electric field; the hot electrons are then injected into the drain-side charge storage node. However, the drain-side region has a low vertical electric field due to the low gate-to-drain potential difference $\left(V_{G D}=V_{G}-V_{D}\right)$. As a result, the injection efficiency is lowered. Moreover, due to high $\mathrm{V}_{\mathrm{D}}$, a Flash memory cell that uses a conventional CHEI method is not suitable for applications with low power operation and high density. In contrast to the conventional device, however, the DSSB device has an abrupt band bending capability near the source-side region, and this capability provides a naturally built-in high lateral electric field that generates sufficient source-side hot electrons, even at a low voltage. In addition, this source-side region experiences a high vertical field due to the high gate-tosource potential difference $\left(\mathrm{V}_{\mathrm{GS}}=\mathrm{V}_{\mathrm{G}}-\mathrm{V}_{\mathrm{S}}, \mathrm{V}_{\mathrm{S}}=\right.$ grounded). As a result, hot electrons are injected into the source-side storage node rather than the drain-side storage node; consequently, the DSSB device has higher injection efficiency than the conventional device. Fig. 3-5(b) shows a simulated energy band diagram for both cases at the programming state. The magnitude of the simulated lateral electric field in a programming state is also shown in Fig. 3-5(c) for different drain voltages. Note that the DSSB FinFET SONOS device has a larger lateral electric field than the conventional FinFET SONOS device under the same programming conditions. This is mainly attributed to the intrinsic sharp band bending of the DSSB junction at the source-side, which is marked by dashed circle in Fig. 3-5(b).

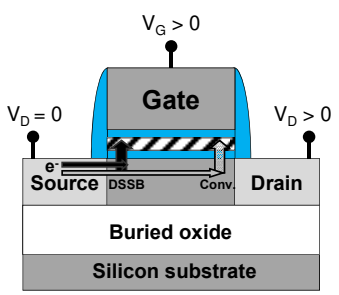

(a)

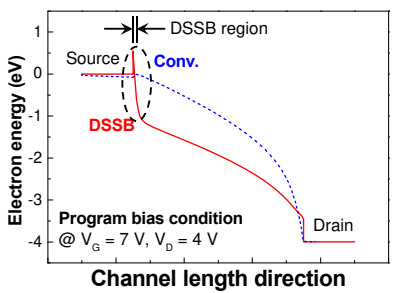

(b)

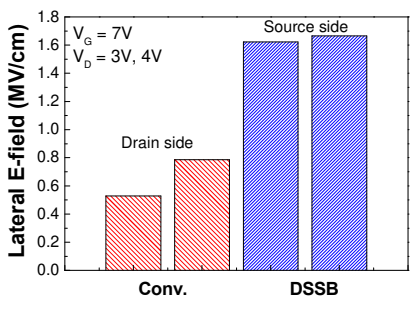

(c)

Fig. 3-5. (a) Comparison of the DSSB device and the conventional device in terms of the charge injection point of hot electrons. (b) Simulated energy band diagrams of both devices at the programming state. The sharp energy band bending should be noted. (c) Simulated lateral electric field for the DSSB device at the source-side and the conventional device at the drain-side. (Choi et al., 2009a)

Fig. 3-6 illustrates the measured programming and erasing transient characteristics. A comparative study was performed with a conventional FinFET SONOS device with the diffused p-n junction as a reference. Under program conditions of $V_{G}=7 \mathrm{~V}$ and $V_{D}=4 \mathrm{~V}$ with $\mathrm{t}_{\mathrm{PGM}}=350 \mathrm{~ns}$, a $\mathrm{V}_{\mathrm{T}}$ shift of approximately $4.5 \mathrm{~V}$ is observed in the DSSB FinFET SONOS device. The DSSB FinFET SONOS device and a conventional FinFET SONOS device for programming show a difference of roughly $3.5 \mathrm{~V}$ in the $\mathrm{V}_{\mathrm{T}}$ shift value at a programming time of $350 \mathrm{~ns}$. This difference is attributed to the high lateral and vertical electric fields at the source-side, which would originate from the sharp band bending caused by the dopant segregated region as well as the intrinsic band profile. In the programming state, electrons injected from the source electrode via thermionic emission or a tunneling process are accelerated by the high lateral electric field and can become hot at the source-side. The 
electrons can subsequently surmount the tunnel oxide barrier around the source-side. As a result, the programming is more efficient. On the other hand, in the erasing state created by FN tunneling, there is no significant difference between the DSSB FinFET SONOS device and the conventional FinFET SONOS device. However, it can be straightforwardly expected that the erasing characteristics can be enhanced by engineering of the gate stack, such as metal-gate (with high workfunction) or bandgap.

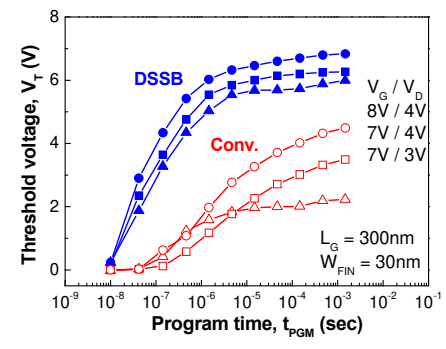

(a)

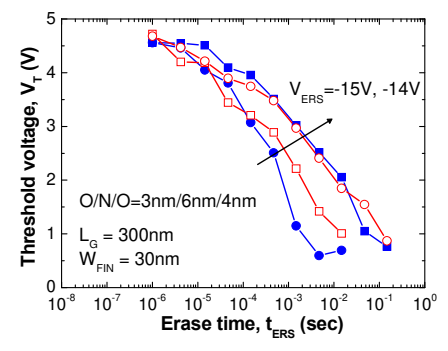

(b)

Fig. 3-6. (a) Program and (b) erase characteristics for DG DSSB and DG conventional SONOS devices (Choi et al., 2009a)

To trace the position of the injected charges experimentally, the transfer characteristics were analyzed after CHEI programming, and the results are shown in Fig. 3-7(a). The observations confirm that hot electrons preferentially inject into the source-side in the DSSB FinFET SONOS device. The behavior of the DSSB FinFET SONOS device is exactly opposite to that of the conventional FinFET SONOS device. After the CHEI programming, the surface potential of the DSSB FinFET SONOS device is more sensitive to $V_{D}$ in the reverse state than in the forward state because of the source-side injection of hot electrons. Even though the $V_{T}$ shift as well as the degradation of the subthreshold swing (SS) caused by captured hot electrons at the drain-side is shown in the forward read state ( $\left.t_{P G M}=320 \mathrm{~ns}\right)$, the amount of captured electrons at the drain-side is much smaller than at the source-side. As a result, the $\mathrm{V}_{\mathrm{T}}$ shift as well as the degradation of SS is not shown in high drain bias of the forward read state. Furthermore, Fig. 3-7(a) shows increased off-state current in relation to $V_{D}$ during the reverse read operation. As shown in Fig. 3-7(b), the simulated energy band diagrams of the forward and reverse read operation are plotted to explain the $V_{T}$ shift and the changed offstate current with varying $V_{D}$ voltage in Fig. 3-7(a). The off-state current in the Schottkybarrier (SB) MOSFET is known to originate from hole tunneling because of the narrowed tunneling width at the drain-side. In a reverse read operation (i.e., the charge trapped region is at the drain-side and read voltage is applied to the drain), the trapped charge can narrow the tunneling width of the drain-side in the off-state. As a result, the off-state current is more sensitive to $V_{D}$ in the reverse read state than in the forward read state; it also increases in relation to the increment of $V_{D}$.

The retention characteristics of the DSSB FinFET SONOS device at various $1 \mathrm{k}$ post-cycled programmed states are illustrated in Fig. 3-8. The characteristics are measured at room temperature. The $\mathrm{V}_{\mathrm{T}}$ window at $\mathrm{t}_{\mathrm{PGM}}=1 \mathrm{~ms}$ is expected to have a value exceeding $4 \mathrm{~V}$ after 10 years.

The drain disturbance of a programmed cell with a relatively high program bias $\left(V_{D}=5 \mathrm{~V}\right)$ was also characterized. In Fig. 3-9, the memory architecture in NOR Flash memory is 


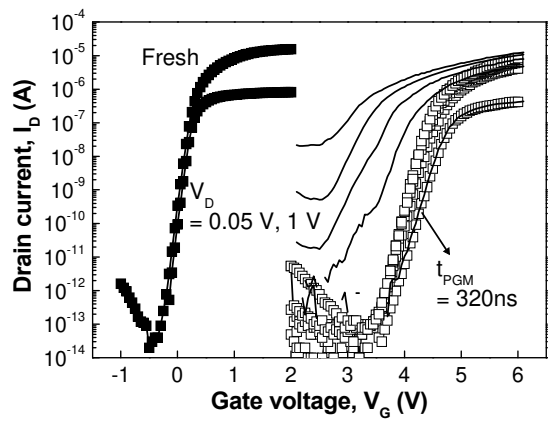

(a)

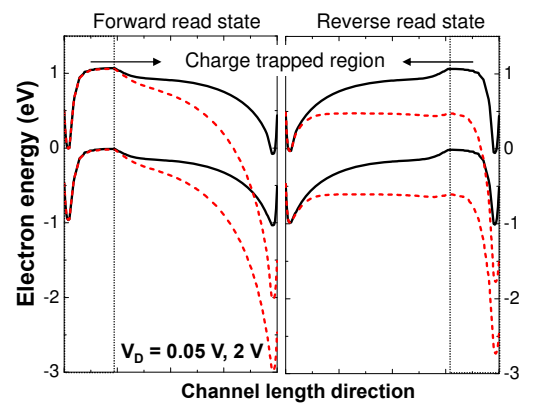

(b)

Fig. 3-7. (a) The $I_{D}-V_{G}$ characteristics as a parameter of $V_{D}$ at the fresh and programmed states in forward and reverse read operations. (b) The simulated energy band diagram of the forward and reverse read state at the off-state. The trapped charges can narrow the tunneling width of the drain-side in the off-state. (Choi et al., 2009b)

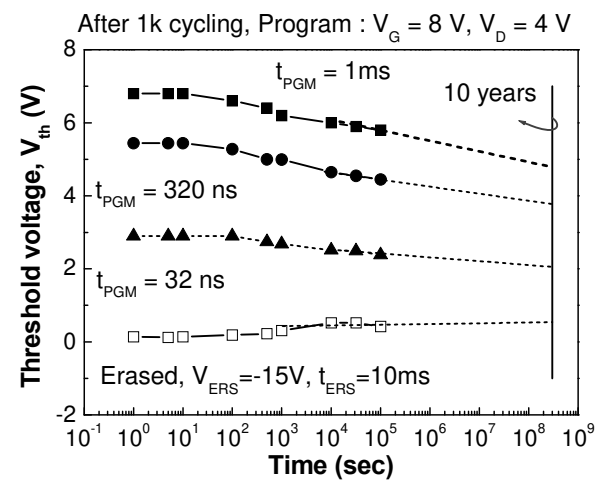

Fig. 3-8. Retention characteristics of a DG DSSB device for MLC in NOR Flash memory operation. (Choi et al., 2009a)

illustrated and the low drain disturbance in DSSB devices is conceptually explained. For the case of cell A (programmed cell), electrons are captured at the source side rather than the drain side. On the other hand, in the case of a conventional device (cell B), trapped electrons at the drain side increase the potential for hot holes to be generated, which results in a drain disturbance (i.e., soft erase). Therefore, improved immunity against drain disturbances is achieved in the DSSB NOR Flash device, as shown in Fig. 3-10. This is primarily due to the trapped electrons located at the source side, as they inhibit hot holes from being injected into the trapped regions.

\subsection{Fowler-Nordheim tunneling program in double-gate DSSB MOSFETs}

One of the advantages of SONOS type Flash memory devices is natural immunity to floating-gate coupling issues, thereby allowing downscaling to the nano-regime. SONOStype devices can operate with very few electrons without displaying erratic behavior. 


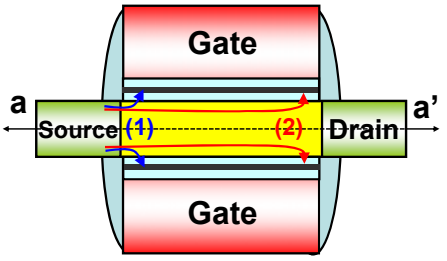

(a)

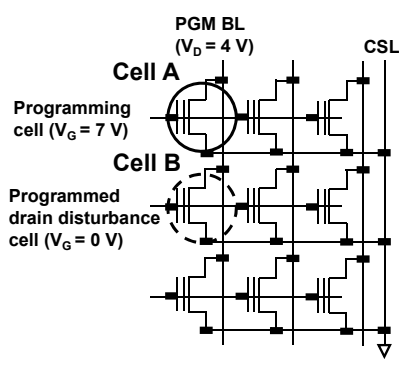

(b)

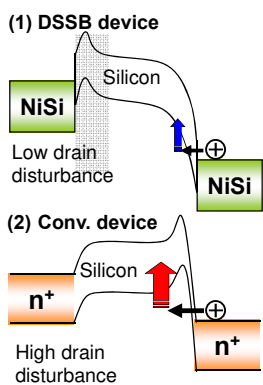

(c)

Fig. 3-9. (a) Schematic of the DG DSSB FinFET SONOS device. (b) Architecture of NOR Flash memory. (c) Conceptually illustrated energy band diagrams at the programmed state for the DSSB and the conventional device. (Choi et al., 2009a)

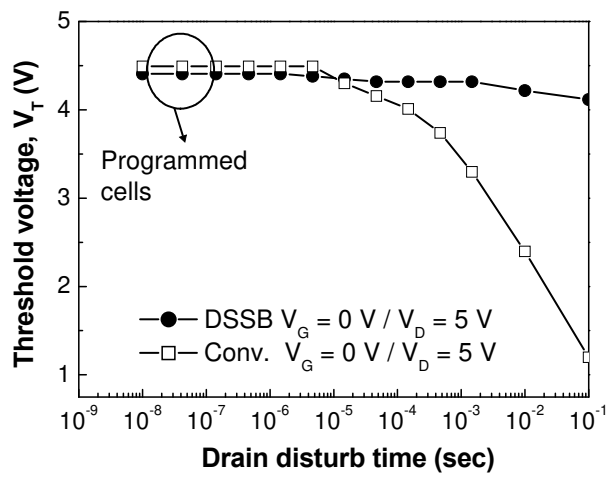

Fig. 3-10. Drain disturbances of DG DSSB and DG conventional devices: Compared to the conventional device, high immunity to drain disturbance is achieved in the DG DSSB device. (Choi et al., 2009a)

However, their programming time is excessively long, falling in a range of $10^{-6} \sim 10^{-3}$ sec due to the Fowler-Nordheim (FN) tunneling mechanism in conventional NAND Flash memory. This makes it difficult for applications requiring high-speed application such as solid-state drive (SSD). In addition, the conventional diffused S/D with deep junctions obstructs further aggressive scaling in the SONOS type memory devices.

Current research on NAND Flash memory is mainly focusing on a 3-D stacking structure realized by deposition of a poly-Si channel. In addition, a junction-free structure, i.e., S/D junctions are not formed, is indispensable, as the formation of S/D junctions is quite difficult due to vertical stacked 3-D Flash memory (Lue et al., 2008). However, this structure cannot be directly applicable to Flash memory with poly-Si channel because of high resistance at the S/D junctions, as aforementioned. Therefore, another method to form S/D junctions is needed. In this section, a novel NAND Flash architecture implemented in the same double-gate DSSB FinFETs SONOS is demonstrated. Fast programming is achieved due to the electrons with extra kinetic energy, i.e., hot carriers, on the dopant segregated 
S/D side. These hot electrons require neither high programming voltage nor long programming time. With the same ground voltage on the S/D junction, hot electrons triggered by sharp energy band bending at the edge of silicided S/D junctions are naturally generated.

\subsubsection{Memory characteristics}

Fig. 3-11(a) explains the operating principle at the programming state. Fast programming is achievable by applying the same ground voltage on both S/D junctions simultaneously (Fig. 3-11(b)). In this case, a locally high lateral of electric field is generated by a sharpened band structure at the dopant-segregated region. Thus, electrons thermally injected or tunneled from S/D edges, i.e., with extra kinetic energy, are energized by this high electric field and are mainly used to program the NAND Flash device. Therefore, fast programming with low voltage is feasible with enhanced tunneling probability. Note that most of trapped electrons in short programming time can be located at the edge of S/D junctions.

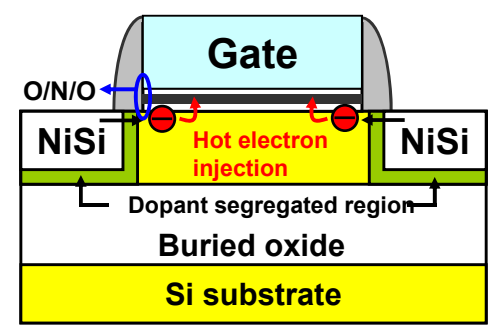

(a)

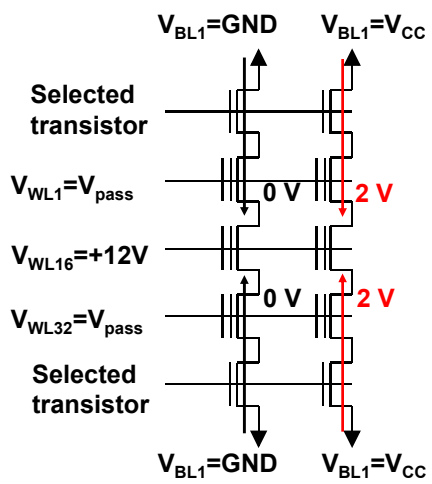

(b)

Fig. 3-11. (a) Schematic of the operating principle of the DSSB FinFET SONOS. (b) The hot electrons energized by the DSSB are used to program for a NAND Flash application by applying the ground voltage on the S/D junctions. (Choi et al., 2009c)

Figs. 3-12(a) and 3-12(b) illustrate the programming and erasing transient characteristics, respectively. As a reference, a conventional FinFET SONOS with a diffused p-n junction is compared. The results demonstrate the excellent program efficiency of the DSSB FinFET SONOS. The program conditions $V_{\mathrm{PGM}}=12 \mathrm{~V}$ with $\mathrm{t}_{\mathrm{PGM}}=100 \mathrm{~ns}$ exhibit a $\mathrm{V}_{\mathrm{T}}$ shift of $4.5 \mathrm{~V}$ in the DSSB FinFET SONOS. It should be noted that a significant $V_{T}$ shift for programming was achieved within a few tens of nanoseconds, which is approximately 1000 times faster than the conventional device. The difference in the $\mathrm{V}_{\mathrm{T}}$ shift between the DSSB FinFET SONOS and a conventional FinFET SONOS for programming was approximately $3 \mathrm{~V}$ at 100 ns programming time. This difference is attributed to hot carrier injection energized by sharp band bending at the dopant segregated S/D junction edge. These outstanding results are among the best results in terms of $\mathrm{V}_{\mathrm{T}}$ window and programming/erasing speed reported to date for FinFET structure flash memory devices. However, there is no significant difference in the erase characteristics of the DSSB FinFET SONOS as compared with a conventional FinFET SONOS because electron de-trapping from the nitride to the silicon substrate is not different between them. 


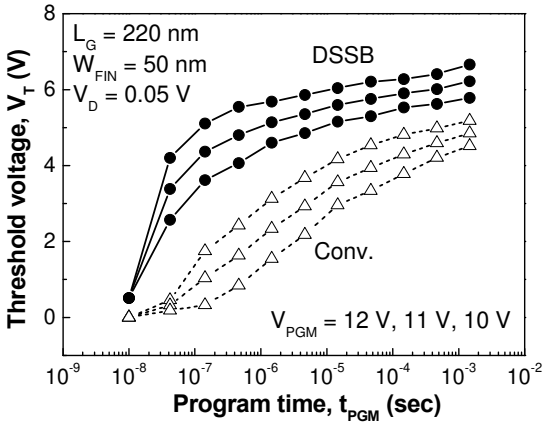

(a)

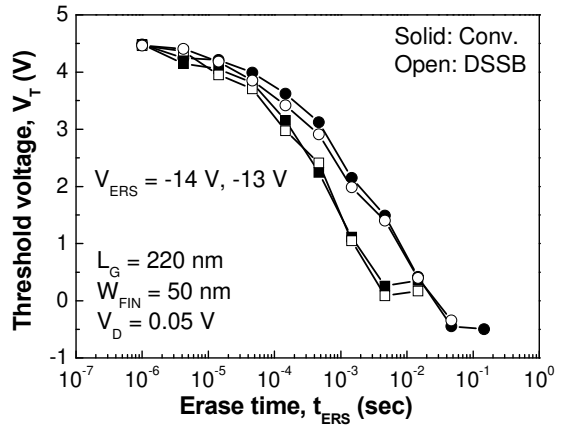

(b)

Fig. 3-12. Program (a) and erase (b) transient characteristics with various program voltages. Excellent program efficiency compared to the control group is achieved due to hot electrons energized by sharp band bending at the S/D. (Choi et al., 2009c)

The tunneling oxide of conventional devices may be non-uniform due to the non-uniform etching profile of the narrow silicon channel; therefore the tunneling probability of electrons at the channel fluctuates significantly in conventional devices. However, for the case of DSSB devices, the trapped electrons are mainly located at the edges of the S/D junction. Therefore, a more parallel $\mathrm{V}_{\mathrm{T}}$ shift can be achieved in the DSSB device. As shown in Fig. 313, a parallel shift among programmed states was found in the DSSB device but not in the conventional device. This implies that two-sided charge injection at the S/D prevails in the DSSB FinFET SONOS device. Note, on the other hand, that an unwanted non-uniform charge injection by FN tunneling occurs in the conventional FinFET SONOS device, resulting in an oblique shift and degradation of the slope.

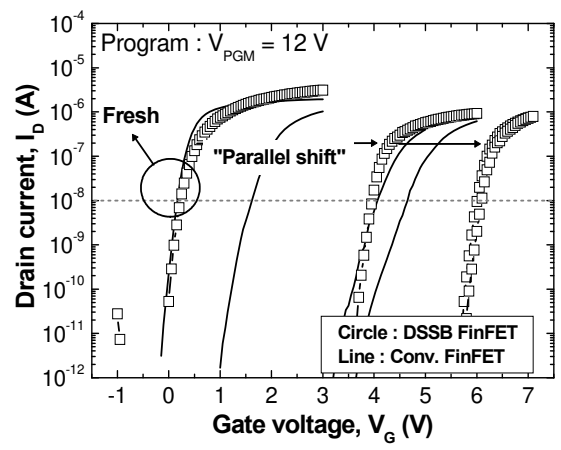

Fig. 3-13. Comparison of the $I_{D}-V_{G}$ shift among various programmed states. A two-sided injected charge produces a parallel shift of I-V. (Choi et al., 2008)

The retention characteristics after $1 \mathrm{k}$ cycling and $\mathrm{P} / \mathrm{E}$ cycling endurance of the DSSB FinFET SONOS were compared to the control group, a conventional FinFET SONOS, and the results are presented in Figs. 3-14 (a) and 3-14(b), respectively. These characteristics were measured at room temperature. Fig. 3-14(a) shows that a rapid degradation of the retention characteristics was monitored during longer programming time. Under this condition the 
stored charges are more likely to be lost due to larger damage by hot carriers, degrading the tunneling oxide quality. Nevertheless, the $\mathrm{V}_{\mathrm{T}}$ margin of the DSSB FinFET SONOS after ten years is larger than that of the conventional device. This is attributed to the high efficiency of programming originating from the sharpened energy band bending by the DSSB structure. $\mathrm{P} / \mathrm{E}$ endurance characteristics are also plotted in Fig. 3-14(b). After $10^{5} \mathrm{P} / \mathrm{E}$ cycles, only a negligible $\mathrm{V}_{\mathrm{T}}$ shift can be seen, thus verifying that the reliability characteristics are satisfactory.

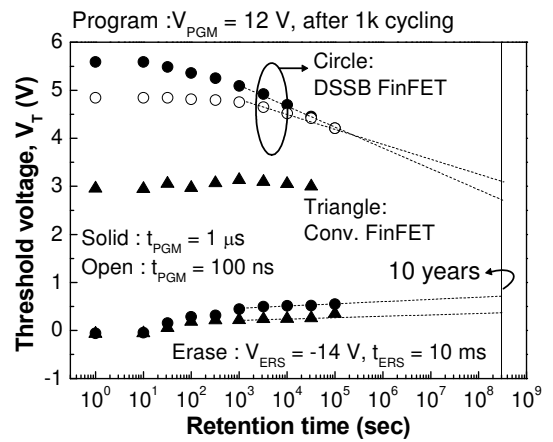

(a)

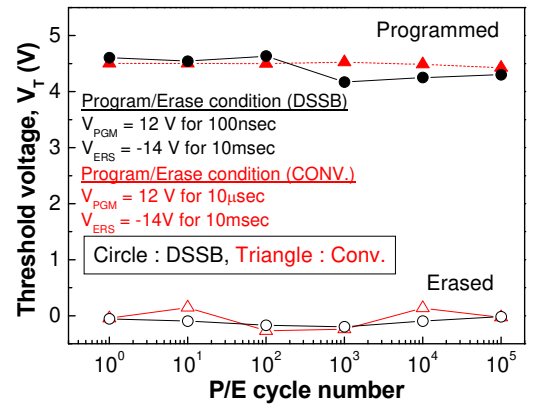

(b)

Fig. 3-14. (a) Post cycling retention comparison of the DSSB FinFET SONOS and a conventional FinFET SONOS. Due to damage of hot electrons, the charge loss of the DSSB FinFET SONOS is larger than that of the conventional device. (b) Measured endurance characteristics of the DSSB FinFET SONOS and the conventional device. A negligible $\mathrm{V}_{\mathrm{T}}$ shift is observed in the P/E states. (Choi et al., 2009c)

\section{Junctionless MOSFETs}

Flash memory has recently scaled rapidly down to a $20 \sim 30 \mathrm{~nm}$ node. However, with researchers relying on conventional approaches, critical scaling limits are being faced, foreshadowing the possibility that further downscaling will eventually be impossible. Hence, a new and innovative device structure is urgently required. Most importantly, among the crucial limitations, the short-channel effects (SCEs) have increasingly become unavoidable technical challenges, as it is difficult to scale the equivalent oxide thickness (EOT) below $10 \mathrm{~nm}$ due to the nature of multi-layered gate dielectrics. Shallow junctions are very important to suppress the SCEs; however, it is difficult to precisely control the junction depth and profile. Moreover, the formation of such shallow junctions becomes a serious concern with 3-dimensional (3D) multi-stacking integration due to the large thermal budget required. For this reason, a "junction-free transistor" based on junction-free virtual S/D for NAND Flash memory was previously reported, and the concept was applied to other types of 3D integrated Flash memory such as Bit Cost Scalable (BiCS) memory (Tanaka et al., 2007), Vertical-Stacked-Array-Transistor (VSAT) memory (Kim et al., 2009), and Terabit Cell Array Transistor (TCAT) memory (Jang et al., 2009), among others (Hubert et al., 2009). However, it can be expected that current flowing through a string of NAND Flash memory will be significantly degraded by pre-existing high resistance regions, i.e., undoped source/drain (S/D) regions, despite that these regions can be transformed into low 
resistance regions via an inversion process by fringing the field from the gate. This can therefore lead to severe back-pattern dependency or result in the failure of read operations. These challenging issues tend to be more severe in 3-D multi-stacked Flash memory where poly-crystalline silicon (poly-Si) is used as a channel (Walker et al., 2009).

Recently, a nanowire transistor known as a "junctionless transistor" or a "gated resistor" was introduced (Colinge et al., 2010). It consists of $\mathrm{n}^{+}$(or $\mathrm{p}^{+}$for a $\mathrm{p}$-channel device) homogenously doped silicon nanowire $(\mathrm{SiNW})$, i.e., an $\mathrm{n}^{+}$source $-\mathrm{n}^{+}$channel $-\mathrm{n}^{+}$drain (or a $\mathrm{p}^{+}$source - $\mathrm{p}^{+}$channel $-\mathrm{p}^{+}$drain) for the $\mathrm{p}$-channel device, with a gate electrode. Junctionless transistors have several advantages compared to traditional inversion-mode transistors: (i) they are easily fabricated; (ii) they are free from S/D junctions therefore have less dopant fluctuation; (iii) they can reduce SCEs; (iv) they can reduce mobility degradation by surface roughness scattering; and (iv) they relax the stringent requirements reducing the gate dielectric thickness. These intrinsic strengths make the concept proposed here attractive for application of a junctionless transistor to Flash memory. However, existing junctionless transistors have an inherent limitation in that they primarily implemented on a SOI wafer.

In this section, an all-around-gate (AAG) junctionless transistor is applied to oxide-nitrideoxide (O/N/O) type charge-trapping Flash memory. By utilizing a deep reactive ion etching (RIE) system (Ng et al., 2009), a junctionless transistor with a suspended SiNW channel with a width of $4 \mathrm{~nm}\left(\mathrm{~W}_{\mathrm{NW}}=4 \mathrm{~nm}\right)$ and a length of $20 \mathrm{~nm}\left(\mathrm{~L}_{\mathrm{G}}=20 \mathrm{~nm}\right)$ is fabricated, where the channel is completely separated from the bulk substrate. The performance is comparable to that of currently reported Flash memory, but it can be scaled down further, below the $20 \mathrm{~nm}$ node, due to the simplified process and the advantages inherited from the junctionless transistor.

\subsection{Operating principle of junctionless MOSFETs}

The operational principle of an n-type junctionless MOSFET is different from that of a standard n-type conventional MOSFET. In the subthreshold region, shown in Fig. 4-1(a), the highly doped channel is fully depleted, and hence it can hold a large electric field. By increasing the gate voltage, the electric field in the channel reduces until a neutral region is created in the center of the channel. At this point, it is possible to define the threshold voltage, because bulk current starts to flow through the center of the channel, as illustrated in Fig. 4-1(b). Then, by further increasing the gate voltage, the depletion width reduces until a completely neutral channel is created, as seen in Fig. 4-1(c). This occurs when the gate voltage equals the flat band voltage. At the onset of this condition, the bulk current reaches its maximum value. Thereafter, by increasing the gate voltage further, negative charges accumulate on the surfaces of the channel, as shown in Fig. 4-1(d). These charges result in surface current, which is similar to the current in a standard n-type conventional MOSFET.

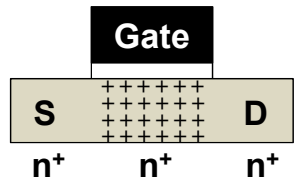

(a)

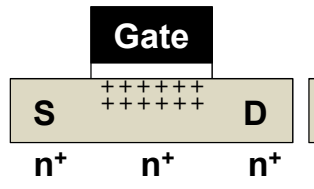

(b)

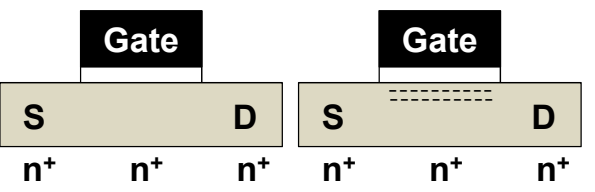

(c)

(d)

Fig. 4-1. (a) Fully depleted channel in subthreshold mode, (b) semi-depleted channel in bulk current mode, (c) flat band mode, and (d) accumulation mode. 


\subsection{Device fabrication}

A (100) bulk silicon wafer is used as a starting material. First, the top of a silicon bulk wafer is uniformly doped by ion implantation with arsenic for the n-channel devices. The implant energies and doses are chosen to yield uniform doping of $2 \times 10^{19} / \mathrm{cm}^{3}$. High doping is required in the junctionless transistor to ensure a high driving current and good source/drain contact resistance. After patterning the active region with $W_{\mathrm{NW}}=30 \mathrm{~nm}$, the Bosch process enabled by the RIE system is employed to form the suspended SiNW separated from the bulk substrate. The suspended SiNW via the Bosch process is achieved by balancing anisotropic etching and passivation steps. Details of the Bosch process can be found in the literature ( $\mathrm{Ng}$ et al., 2009). The scanning electron microscopy (SEM) images in Fig. 4-2 clearly show the suspended SiNWs. The gap distance between the SiNW and bulk substrate is approximately $250 \mathrm{~nm}$. After the formation of the SiNWs, channel stop implantation with boron ions is applied. Subsequently, two iterations of sacrificial oxidation are employed for further reduction of the width $\left(\mathrm{W}_{\mathrm{NW}}=4 \mathrm{~nm}\right)$ of the SiNW and to make the channel smooth, followed by the formation of shallow trench isolation (STI). Next, an $\mathrm{O} / \mathrm{N} / \mathrm{O}$ layer with a thickness of $2.8 \mathrm{~nm} / 6.2 \mathrm{~nm} / 7 \mathrm{~nm}$ (using a thermal oxide and LP-CVD nitride/TEOS oxide) and an in-situ $\mathrm{n}^{+}$poly-Si gate (using LP-CVD poly-Si) are formed sequentially. Afterwards, a gate length $\left(\mathrm{L}_{\mathrm{G}}\right)$ of $20 \mathrm{~nm}$ is patterned. Horizontal and vertical transmission electron microscopy (TEM) images of the fabricated junctionless transistor are also shown in Fig. 4-2.

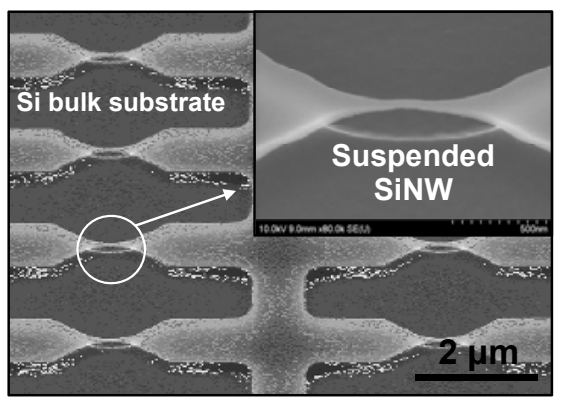

(a)
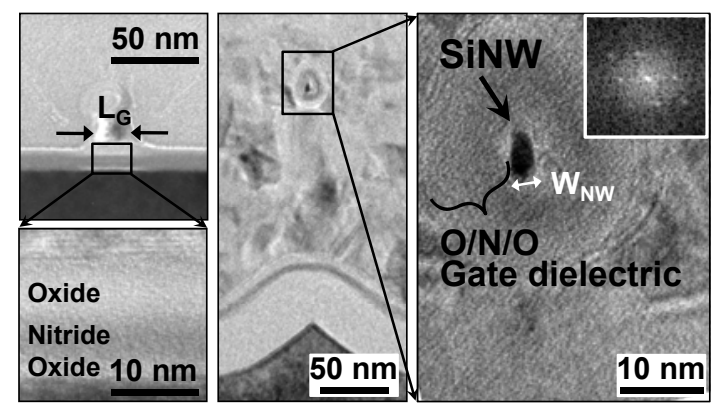

(b)

Fig. 4-2. (a) SEM image and magnified views of the suspended SiNW on the bulk substrate and (b) Horizontal and vertical TEM images in the $\mathrm{L}_{\mathrm{G}}$ direction in the AAG junctionless transistor with the $\mathrm{O} / \mathrm{N} / \mathrm{O}$ gate dielectric. The width $\left(\mathrm{W}_{\mathrm{NW}}\right)$ and length $\left(\mathrm{L}_{\mathrm{G}}\right)$ of the SiNW channel are approximately $4 \mathrm{~nm}$ and $20 \mathrm{~nm}$, respectively. The thickness of the O/N/O layers for the charge storage node is $2.8 \mathrm{~nm} / 6.2 \mathrm{~nm} / 7 \mathrm{~nm}$. (Choi et al., 2011)

\subsection{Memory characteristics}

Fig. 4-3(a) shows the P/E transient characteristics of junctionless AAG SONOS devices with a $20 \mathrm{~nm} \mathrm{~L}_{\mathrm{G}}$ and a $4 \mathrm{~nm} \mathrm{~W}_{\mathrm{NW}}$ for various $\mathrm{P} / \mathrm{E}$ conditions. A large $\mathrm{P} / \mathrm{E}$ window $\left(\Delta \mathrm{V}_{\mathrm{T}}\right)$ up to $6.5 \mathrm{~V}$ was attained with the aid of a GAA structure despite the highly scaled device size, demonstrating the cell suitability for MLC operations. No erase saturation phenomenon was observed, even at $-15 \mathrm{~V}$, despite the $\mathrm{n}^{+}$poly-Si gate. This indicates that there is no need to use a metal-gate or high-k blocking oxide. Moreover, as seen in Fig. 4-3(b), program inhibition is 
achieved by direct raising of the unselected bit-line potential. It is implicit that there is no need to introduce a complex self-boosting method. A high incremental step pulse program (ISPP) slope (0.7) is also attained, even with a $L_{G}$ value of $20 \mathrm{~nm}$.

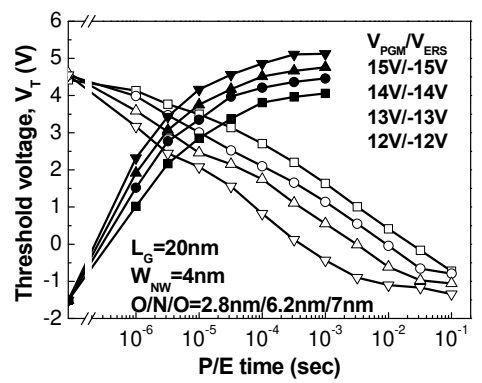

(a)

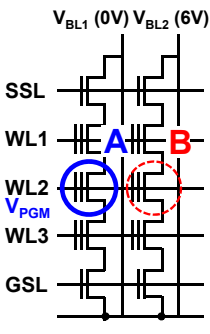

(b)

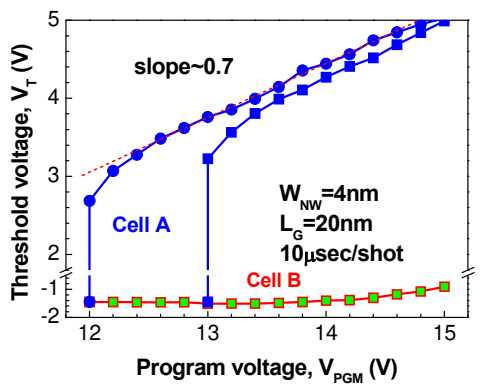

(c)

Fig. 4-3. (a) Program and erase transient characteristics of the junctionless AAG SONOS device. (b) ISPP programming and related inhibit method. Program inhibition is achieved by directly raising the unselected bit-line potential. For a programmed cell, a higher incremental step pulse program (ISPP) slope is also attained, even at $20 \mathrm{~nm} \mathrm{LG}$. (Choi et al., 2011)

3D NAND structures with a floating body require careful consideration when designing S/D junctions for enhanced erase characteristics. To fix the floating body potential during erase operations effectively, a sufficient number of holes must be generated by band-to-band tunneling from the S/D junctions. Therefore, the S/D junctions need to be heavily doped, abrupt, and uniform. Unless 3D NAND structures satisfy the aforementioned demands, uniform and efficient erase characteristics cannot be ensured in conventional diffused S/D and junction-free virtual S/D structures (Figs. 4-3(a) and 4-3(b)). Fig. 4-3(c) compares the distribution of the erased $V_{T}$ for the junctionless and inversion-mode AAG SONOS devices. Contrary to the inversion-mode devices, the S/D of junctionless devices is precisely controlled by the gate electric field. As a result, a uniformly distributed erased $V_{T}$ is successfully obtained without any $\mathrm{V}_{\mathrm{T}}$ correction methods.

Because the conduction of a junctionless device initially occurs in the center of an $\mathrm{n}^{+}$-doped SiNW channel, the device can be less sensitive to the interface trap generated from $\mathrm{P} / \mathrm{E}$ cycles compared to a conventional inversion-mode device, as shown in Fig. 4-4. In a TCAD simulation, it is confirmed that the acceptor-type interface trap does not significantly affect the $\mathrm{V}_{\mathrm{T}}$ shift in a junctionless device. Note that the higher the doping concentration of a SiNW channel is, the stronger the $\mathrm{P} / \mathrm{E}$ endurance becomes. Moreover, reasonable post-cyclic data retention characteristics were achieved.

\section{Conclusions}

In this chapter, as we confront challenges of current Flash memory technology and as the design rule deviates from the historical scaling paradigm, a new type of Flash memory cell based on the structure of dopant-segregated Schottky-barrier (DSSB) MOSFETs, which has an ultra-thin pocket layer with high-dose dopants surrounding the interface between the 


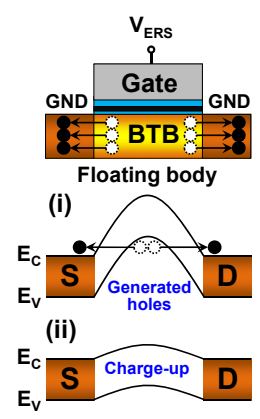

(a)

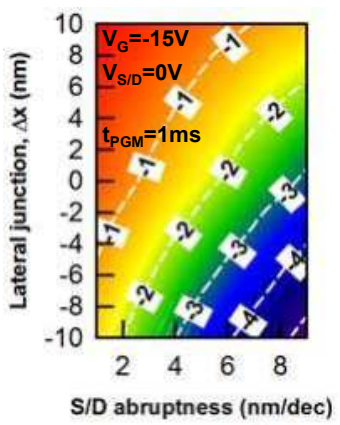

(b)

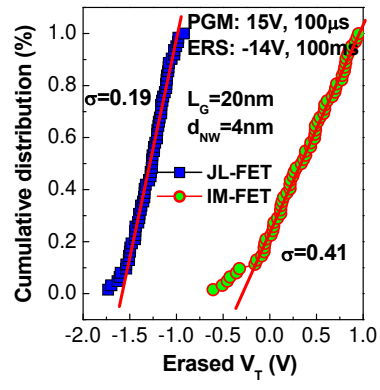

(c)

Fig. 4-3. (a) Erase operations for the 3D NAND structure with a floating body: (i) First, the floating body potential follows the gate potential $\left(\mathrm{V}_{\mathrm{ERS}}\right)$. As a result, holes are generated by band-to-band tunneling. (ii) Second, the generated holes can pin the floating body potential. (b) TCAD simulation of the floating body potential during the erase operation. (c) Distribution of erased $\mathrm{V}_{\mathrm{T}}$ values for junctionless and inversion-mode AAG SONOS devices. (Choi et al., 2011)

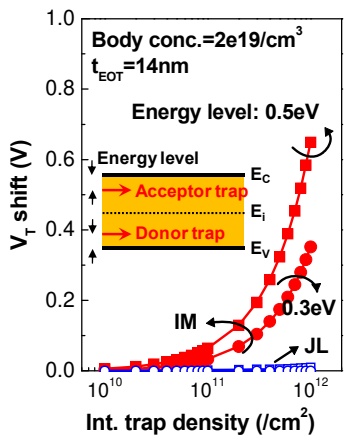

(a)

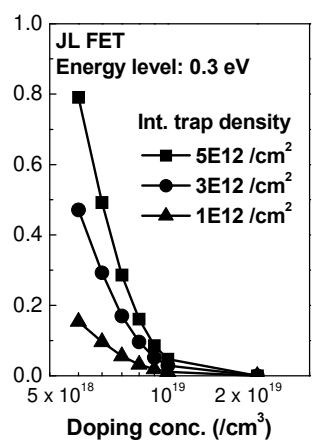

(b)

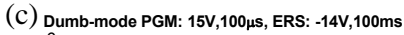
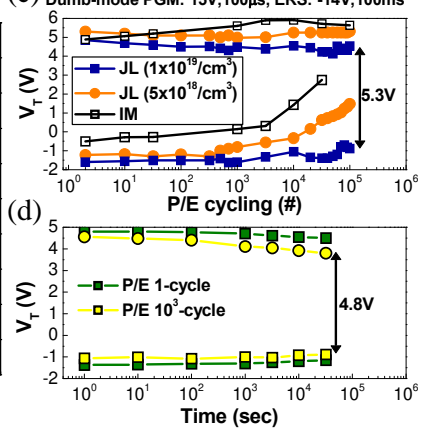

Fig. 4-4. (a) Simulated $\mathrm{V}_{\mathrm{T}}$ shift versus interface trap density $\left(\mathrm{N}_{\mathrm{it}}\right)$ as a parameter of the energy level of both acceptor- and donor-type traps. (b) Simulated $\mathrm{V}_{\mathrm{T}}$ shift versus doping concentration of the SiNW channel in the junctionless device. (c) Dumb-mode P/E cycling (without any P/E verify) endurance test. (d) Post-cycling retention characteristics of the junctionless device. (Choi et al., 2011)

metallic silicide material for source/drain (S/D) and the channel, is proposed. The hot carriers intrinsically generated from the shallow DSSB S/D junctions can be utilized for the advancement of both the NAND and the NOR type Flash memory cell. With the aid of hot carriers that can be generated by elevated electric field at the DSSB S/D junctions stemming from the abrupt band bending, the probability to be trapped into a charge storage node of Flash memory, such as polysilicon layer in the floating gate memory device or the nitride layer in the SONOS memory device, is enhanced. Therefore, the DSSB MOSFET shows very fast programming time at low programming voltage, compared to conventional MOSFET 
based on p-n S/D junctions. Besides, the superior scalability resulting from the abrupt and shallow junctions can also be achieved without the constraint of the parasitic resistance due to metallic silicided material. Therefore, the DSSB devices can be a premier choice for future nano-electronics applications of the logic and Flash memory device since they do not only enable continuation of device scaling due to the improved electrostatics but also provide benefits for an alternative memory cell.

Moreover, a highly scaled AAG junctionless transistor SONOS memory cell with acceptable $\mathrm{P} / \mathrm{E}$ behaviors, cycling endurance, and data retention is also demonstrated. The junctionless transistor memory cell inherited the scaling advantages of not only the AAG structure but also the junctionless transistor. Therefore, the junctionless transistor memory cell, together with DSSB MOSFETs, is an excellent candidate for the next-generation 3-D NAND Flash memory.

\section{Acknowledgment}

This work was supported by the IT R\&D program of MKE/KEIT [10035320, Development of novel 3D stacked devices and core materials forthe next generation flash memory], the National Research Foundation (NRF) grant funded by the Korea government (No. K20901000002-09E0100-00210), Nano R\&D program through the National Research Foundation of Korea funded by the Ministry of Education, Science, and Technology (grant number : 2009-0082583), and Samsung Electronics Co., Ltd.

\section{References}

Walker, A. J. (2009). Sub-50-nm dual-gate thin-film transistors for monolithic 223 3-D Flash, IEEE Transaction on Electron Devices, Vol. 56, No. 11, pp. 2703-2710, IEEE, ISSN:0018-9383

Larson, J. M. et al. (2006). Overview and Status of Metal S/D Schottky-Barrier MOSFET Technology, IEEE Transaction on Electron Devices, Vol. 53, No. 5, pp. 1048-1058, IEEE ISSN:0018-9383

Kinoshita, A. et al. (2004). Solution for High-Performance Schottky-Source/Drain MOSFETs: Schottky Barrier Height Engineering with Dopant Segregation Technique, IEEE VLSI Symp. Tech. Dig., pp. 168-169, IEEE, ISBN:4-900784-00-1

Muraka, S. P. et al. (1987). Dopant redistribution in silicide-silicon and silicidepolycrystalline silicon bilayered structures, Journal of Vacuum Science E Technology, Vol. 5, No. 6, pp. 1674-1688, ISSN:1071-1023

Uchida, K. et al. (2000). Enhancement of hot-electron generation rate in Schottky source metal-oxide-semiconductor field-effect transistors, Applied Physics Letters, Vol. 76, No. 26, pp. 3992-3994, ISSN:0003-6951

Kinoshita, A. et al. (2006). Comprehensive Study on Injection Velocity Enhancement in Dopant-Segregated Schottky MOSFETs, IEEE IEDM Tech. Dig., pp. 1-4, IEEE, ISBN:978-1-4244-237-4

$\mathrm{Wu}, \mathrm{A}$. T. et al. (1986). A novel high-speed, 5-volt programming EPROM structure with source-side injection, IEEE IEDM Tech. Dig., pp. 584-587, IEEE, ISBN:978-1-4244237-4 
Choi, S.-J. et al. (2008). High Speed Flash Memory and 1T-DRAM on Dopant Segregated Schottky Barrier (DSSB) FinFET SONOS Device for Multi-functional SoC Applications, IEEE IEDM Tech. Dig., pp. 223-226, IEEE, ISBN:978-1-4244-237-4

Choi, S.-J. et al. (2009a). Performance Breakthrough in NOR Flash Memory with DopantSegregated Schottky-Barrier (DSSB) SONOS Devices, IEEE VLSI Symp. Tech. Dig., pp. 222-223, IEEE, ISBN:4-900784-00-1

Choi, S.-J. et al. (2009b). High Injection Efficiency and Low-Voltage Programming in a Dopant-Segregated Schottky Barrier (DSSB) FinFET SONOS for NOR-type Flash Memory, IEEE Electron Device Letters, Vol. 30, No. 3, pp. 265-268, IEEE, ISSN:07413106

Lue, H.-T. et al. (2008). A novel junction-free BE-SONOS NAND Flash, IEEE VLSI Symp. Tech. Dig., pp. 140-141, IEEE, ISBN:4-900784-00-1

Choi, S.-J. et al. (2009c). Enhancement of Program Speed in Dopant-Segregated SchottkyBarrier (DSSB) FinFET SONOS for NAND-Type Flash Memory, IEEE Electron Device Letters, Vol. 30, No. 1, pp. 78-81, IEEE, ISSN:0741-3106

Tanaka, H. et al. (2007). Bit cost scalable technology with punch and plug process for ultra high density Flash memory, IEEE VLSI Symp. Tech. Dig., pp. 14-15, IEEE, ISBN:4900784-00-1

Kim, J. et al. (2009). Novel vertical-stacked-array-transistor (VSAT) for ultra-high-density and cost-effective NAND Flash memory devices and SSD (solid state drive), IEEE VLSI Symp. Tech. Dig., pp. 186-187, IEEE, ISBN:4-900784-00-1

Jang, J. et al. (2009). Vertical cell array using TCAT (terabit cell array transistor) technology for ultra high density NAND Flash memory, IEEE VLSI Symp. Tech. Dig., pp. 192193, IEEE, ISBN:4-900784-00-1

Hubert, A. et al. (2009). A stacked SONOS technology, up to 4 levels and $6 \mathrm{~nm}$ crystalline nanowires, with gate-all-around or independent gates ( $\Phi$-Flash), suitable for full 3D integration, IEEE IEDM Tech. Dig., pp. 637-640, IEEE, ISBN:978-1-4244-237-4

Colinge, J.-P. et al. (2010). Nanowire transistors without junctions, Nature Nanotechnology, Vol. 5, pp. 225-229, ISSN: 1748-3387

Ng, R. M. Y. et al. (2009). Vertically Stacked Silicon Nanowire Transistors Fabricated by Inductive Plasma Etching and Stress-Limited Oxidation, IEEE Electron Device Letters, Vol. 30, No. 5, pp. 520-522, IEEE, ISSN:0741-3106

Choi, S.-J et al. (2011) A Novel Junctionless All-Around-Gate SONOS Device with a Quantum Nanowire on a Bulk Substrate for 3D Stack NAND Flash Memory, IEEE VLSI Symp. Tech. Dig., in press, IEEE, ISBN:4-900784-00-1 


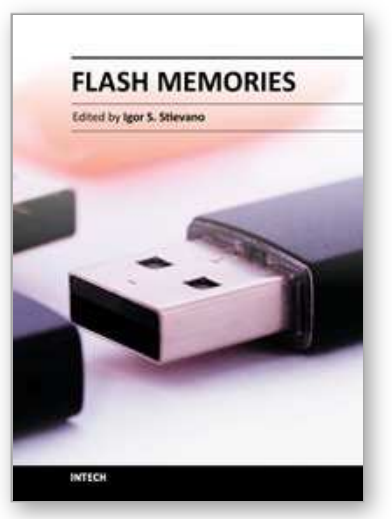

\author{
Flash Memories \\ Edited by Prof. Igor Stievano
}

ISBN 978-953-307-272-2

Hard cover, 262 pages

Publisher InTech

Published online 06, September, 2011

Published in print edition September, 2011

Flash memories and memory systems are key resources for the development of electronic products implementing converging technologies or exploiting solid-state memory disks. This book illustrates state-ofthe-art technologies and research studies on Flash memories. Topics in modeling, design, programming, and materials for memories are covered along with real application examples.

\title{
How to reference
}

In order to correctly reference this scholarly work, feel free to copy and paste the following:

Sung-Jin Choi and Yang-Kyu Choi (2011). Source and Drain Junction Engineering for Enhanced Non-Volatile Memory Performance, Flash Memories, Prof. Igor Stievano (Ed.), ISBN: 978-953-307-272-2, InTech, Available from: http://www.intechopen.com/books/flash-memories/source-and-drain-junction-engineering-for-enhancednon-volatile-memory-performance

\section{INTECH}

open science | open minds

\author{
InTech Europe \\ University Campus STeP Ri \\ Slavka Krautzeka 83/A \\ 51000 Rijeka, Croatia \\ Phone: +385 (51) 770447 \\ Fax: +385 (51) 686166 \\ www.intechopen.com
}

\author{
InTech China \\ Unit 405, Office Block, Hotel Equatorial Shanghai \\ No.65, Yan An Road (West), Shanghai, 200040, China \\ 中国上海市延安西路65号上海国际贵都大饭店办公楼405单元 \\ Phone: +86-21-62489820 \\ Fax: +86-21-62489821
}


(C) 2011 The Author(s). Licensee IntechOpen. This chapter is distributed under the terms of the Creative Commons Attribution-NonCommercialShareAlike-3.0 License, which permits use, distribution and reproduction for non-commercial purposes, provided the original is properly cited and derivative works building on this content are distributed under the same license. 